\title{
Coeficiente de fragilidad como medida más representativa de la resistencia a la abrasión de pavimentos cerámicos
}

\author{
M. ROMERO ${ }^{(*)}$, J. $^{\text {a }}$ RINCON ${ }^{(*)}$, A. R. BOCCACCINI ${ }^{* *}$ \\ (*) Lab. de Materiales Vitrocerámicos, Inst. E.Torroja Ciencias de la Construcción, CSIC, Madrid \\ ${ }^{(* *)}$ Departamento de Materiales, Imperial College, Londres, Reino Unido
}

\begin{abstract}
La determinación de la resistencia a la abrasión de pavimentos cerámicos es objeto actualmente de una amplia polémica tanto científica como tecnológica, que afecta lógicamente a la aceptación por parte del sector industrial de mejores métodos para determinar dicha propiedad en pavimentos y revestimientos de tipo cerámico. Aunque el método tradicional PEI (Porcelain Enamel Institute) es aceptado generalmente por los fabricantes de azulejos; no obstante, es objeto de numerosas críticas debido a sus lagunas de tipo científico para la interpretación de resultados. Por tanto, diferentes investigadores están en estos momentos proponiendo métodos alternativos a dicho método PEI o complementarios, que permitan una evaluación más fidedigna de los resultados tanto entre los materiales que existen en el mercado, como los de tipo experimental que se obtienen en investigaciones en curso de realización. Se propone, pues, un método alternativo al del PEI, demostrándose que introduciendo el concepto de fragilidad como el cociente entre la microdureza y la tenacidad $\left(\mathrm{B}=\mathrm{H}_{\mathrm{V}} / \mathrm{K}_{\mathrm{IC}}\right)$, determinados a partir de ensayos de indentación con puntas Vickers y Knopp, es posible encontrar una correlación al menos semicuantitativa entre dicho parámetro y las escalas tradicionales de resistencia a la abrasión de pavimentos y revestimientos cerámicos.
\end{abstract}

Palabras clave : azulejos, pavimentos cerámicos,ensayos de indentación, ensayos abrasión, fragilidad

\section{Fragility index as more efficient measurement for determination of abrasion resistance of ceramic tiles}

The abrasion resistance on ceramic tiles nowadays is focus of high interest and scientific and technological criticism affecting the standardization. Though the PEI (Porcelain Enamel Institute) method is generally accepted by manufacturers this is object of strong discussion due to lack of scientific control of final stage of material after abrasion tests. Nevertheless, this method is generally accepted and included in the commercial information of products. Therefore, several investigations are being carried out in order to improve this method and to find alternative procedures to PEI method, both for commercial and experimental products. Here is shown that,introduction of fragility concept as $\mathrm{B}=\mathrm{H}_{\mathrm{V}} / \mathrm{K}_{\mathrm{IC}}$ determined from indentation tests with Vickers and Knopp diamond indenters in a wide range of representative tile products,is possible to see some correlation with abrasion resistance of ceramic tiles determined with PEI method.

Palabras clave : tiles, ceramic pavements, indentations tests, fragility, abrasion resistance

\section{INTRODUCCIÓN}

La producción de pavimentos y revestimientos cerámicos alcanza en estos momentos un valor espectacular en millones de $\mathrm{m}^{2}$ y en nuevos productos con un alto valor económico a nivel mundial motivado por un mercado cada vez más exigente. De todas las propiedades que se exigen a una plaqueta de pavimento, y especialmente en el caso de los de tipo cerámico, es su resistencia a la abrasión superficial a la que se exigen cada vez mayores prestaciones y que permite comparar mejor el comportamiento al desgaste entre diferentes tipos de productos que actualmente existen en el mercado: gres rústico, gres vidriado, gres porcelánico (mate o pulido), gres porcelánico vidriado $\mathrm{y}$, por último, los de recubrimientos de tipo vitrocerámico. Usualmente, los fabricantes del sector aceptan la resistencia a la abrasión determinada por el método PEI (Porcelain Enamel Institute), transferida hace tiempo desde su uso en esmaltes sobre metal, y que consiste en determinar la pérdida del brillo de la superficie en función de las revoluciones que soporta la misma, sometida a desgaste en húmedo por una distribución uniforme de bolas de acero y polvo de alúmina $(1,2)$. Pero este procedimiento adolece de innumerables dificultades para permitir una evaluación científica y realmente comparativa entre la amplia gama de productos que existen y que afloran al mercado continuamente. Otro inconveniente añadido en el uso de este procedimiento es la dificultad de distinguir entre piezas con niveles de PEI intermedios, ya que en mismos grados de resistencia (como en el PEI II y PEI III) se incluyen con resistencias a revoluciones en un amplio margen y, sobre todo, por la no definición en la Norma correspondiente (1) en cuanto al modo de determinar las variaciones de brillo de una manera cuantitativa.

Desde que los métodos de indentación en la caracterización de propiedades mecánicas de materiales se generalizaron por su comodidad de aplicación y por su facilidad para comparar el comportamiento entre materiales, se vienen usando dichos métodos en materiales cerámicos avanzados debido a que permiten una mejor determinación de las propiedades mecánicas entre diversos productos, siendo Lawn y Marshall (3) los primeros que relacionaron la tenacidad con el concepto de fragilidad. Más recientemente, se ha incorporado el uso de los métodos de indentación en diversos tipos de pavimentos cerámicos $(4,5)$. Desde hace unos años se vienen realizando numerosos intentos por encontrar una comparación cuantitativa entre la resistencia a la abrasión y la textura superficial (rugosidad) (6) o midiendo el brillo por un método cuantita- 
tivo y no meramente subjetivo como hasta ahora establece la correspondiente Norma EN 154 (1).

Hasta el momento no se ha encontrado al menos un modelo o una correlación física de utilidad que permita establecer una relación definitiva entre la resistencia a la abrasión y las medidas realizadas por indentación, aunque este intento se ha iniciado hace unos años para materiales cerámicos avanzados $(7,8)$. Por tanto, el objetivo de esta investigación es estudiar una amplia gama de pavimentos comercializados procedentes de diversos fabricantes, realizando ensayos de indentación para obtener tanto la microdureza como la tenacidad de fractura. Aplicando el concepto de fragilidad como la relación : B $=\mathrm{H}_{\mathrm{V}} / \mathrm{K}_{\mathrm{IC}}$, ya mencionado por Moreno y col. en 1994 (4) y readaptado por Boccaccini $(7,8)$, el objetivo de este trabajo ha sido relacionar dicho parámetro con los datos de resistencia a la abrasión por el método PEI obtenido en las mismas muestras de pavimentos cerámicos.

\section{MATERIALES Y MÉTODOS}

Partiendo de 20 tipos diferentes de pavimentos cerámicos tanto en productos vidriados (con espesor de vidriado entre 0,3 y $0,5 \mathrm{~mm}$ ) como en no- vidriados que se producen en el área de Castellón, se han realizado ensayos previos de indentación con un microdurómetro Matzusawa para determinar las condiciones óptimas de carga y duración de la misma, así como las posibilidades de obtención de medidas fiables en su superficie tanto en productos vidriados como no vidriados. A partir de estos ensayos previos se han seleccionado un total de 11 muestras que representan a todos los tipos de productos ensayados. Todos los ensayos se han realizado con punta cuadrangular de diamante tipo Vickers calculándose los valores de Microdureza $\left(\mathrm{H}_{\mathrm{V}}\right)$ y Tenacidad de fractura $\left(\mathrm{K}_{\mathrm{IC}}\right)$ por los métodos ya habituales en materiales vítreos (4) utilizando una carga de $1000 \mathrm{~g}(9,81 \mathrm{~N})$ con un tiempo de aplicación de la misma de $15 \mathrm{~s}$. En cada superficie de los materiales investigados se ensayaron hasta 10 indentaciones elegidas en zonas al azar, a partir de las cuales se ha calculado la media aritmética que es el valor que se ha tomado para su comparación con la escala del método PEI (Porcelain Enamel Institute) determinado de acuerdo con la Norma ISO correspondiente (1).

A partir de los datos de indentación que permiten obtener $\mathrm{H}_{\mathrm{V}}$ y $\mathrm{K}_{\mathrm{IC}}$ se ha calculado el "índice de fragilidad" como la relación : $\mathrm{B}=\mathrm{H}_{\mathrm{V}} / \mathrm{K}_{\mathrm{IC}}$. Este concepto tiene en cuenta la idea básica de que dicho índice mide la respuesta de un material a dos fuerzas competitivas como son la deformación por indentación y la fractura manifestada por la propagación de grietas; de manera que en el caso de un acero de resistencia media se obtiene un valor de $\mathrm{B}=0,1 \mu \mathrm{m}^{-1 / 2}$, mientras que en un monocristal de Silicio se obtiene un valor de $B=17 \mu m^{-1 / 2}$ (8). Según Sehgal y col. (9) dicho índice se puede estimar por la expresión : $\mathrm{B}=\alpha_{0}{ }^{-3 / 4} \mathrm{H}^{3 / 4} \mathrm{E}^{-1 / 2} \gamma \mathrm{P}^{-1 / 4}(\mathrm{c} / \mathrm{a})^{3 / 2}$, siendo $\mathrm{E}$ el módulo de elasticidad, c y a las longitudes de las diagonales de la grieta y de la indentación de punta piramidal tipo Vickers. $\mathrm{H}$ es el valor de microdureza Vickers y P es la carga aplicada. Tanto $\alpha_{0}$ como $\gamma$ son dos parámetros : el primero es de tipo geométrico y depende del indentador, siendo 2,15 para el de tipo Vickers y el segundo es empírico y adimensional. En el caso de materiales vítreos un valor de $\gamma=18$ da lugar a un buen ajuste de los datos experimentales del índice de fragilidad tal y como se ha comprobado en (7).

\section{RESULTADOS Y DISCUSIÓN}

En la Tabla I se muestran los valores obtenidos para los materiales aquí investigados tanto de la microdureza como de la tenacidad de fractura o constante crítica de intensidad de tensiones. A partir de dichas propiedades se ha calculado la relación que permite tener los valores del índice de fragilidad de las superficies examinadas. Se ha comprobado que los valores de fragilidad (B) están en el intervalo : 2,3- 5,1 $\mu \mathrm{m}^{-1 / 2} \mathrm{y}$ que a su vez se correlacionan con las relaciones (c/a) de la longitud de grieta a semidiagonal de las huellas de indentación. Se han desarrollado métodos de cálculo para una estimación teórica de la fragilidad en el caso de materiales cerámicos avanzados masivos (8), como por ejemplo en el caso de materiales compuestos con placas de alúmina dispersa en matrices de vidrios de borosilicato o de aluminosilicato de calcio, comprobándose hasta ahora que los valores de índice de fragilidad para este tipo de materiales están en el intervalo : $1-13 \mu \mathrm{m}^{-1 / 2}$. Se observa que dicho parámetro disminuye su valor cuando es más alto el valor que se alcanza en la escala de PEI (desde I a V) de resistencia a la abrasión para cada material. Así pues, los valores de resistencia mecánica a la abrasión se corresponden con valores menores de dicho parámetro de fragilidad. Si se examina con más detalle este hecho se comprueba que al aumentar el valor en la escala de resistencia a la abrasión (PEI), se aprecia que al mismo tiempo el valor del parámetro B de fragilidad disminuye (Fig. 1). Otros autores han encontrado correlaciones similares pero con parámetros geométricos como es la Resistencia al Desgaste Profundo (rev. $\mu \mathrm{m} / \mu \mathrm{m}^{2}$ ) a partir de la Rugosidad determinada con un rugosímetro (5), pero dicho método es más complejo y requiere disponer de un rugosímetro. En cualquier caso, con los métodos citados aun no se ha llegado a una formulación teórica del comportamiento frente al desgaste por abrasión de materiales de pavimentos cerámicos.

Tanto si se representa los valores obtenidos de microdureza como los de tenacidad frente a las revoluciones que soporta el material en el ensayo de abrasión tipo PEI, se ha comprobado que apenas varían estas propiedades con la escala obtenida para dicho ensayo, por lo que el fenómeno es más complejo de lo que se pretende, observándose únicamente una peque-

TABLA I: VALORES DE INDICE DE FRAGILIDAD, MICRODUREZA Y TENACIDAD EN RELACION CON VALORES PEI DE RESISTENCIA A LA ABRASIÓN DE UNA SERIE DE PAVIMENTOS CERÁMICOS COMERCIALES

\begin{tabular}{|c|c|c|c|c|c|}
\hline $\begin{array}{c}\text { Clase } \\
\text { PEI }\end{array}$ & $\begin{array}{c}\text { Revoluciones } \\
(\mathbf{a b r a s i o ́ n} \\
\mathbf{v i s i b l e})\end{array}$ & Muestra & $\begin{array}{c}\mathbf{B}\left(\mu \mathrm{m}^{-1 / 2}\right) \\
( \pm \sigma=0,1)\end{array}$ & $\begin{array}{c}\mathbf{H}_{\mathbf{v}}(\mathrm{GPa}) \\
( \pm \sigma=0,05)\end{array}$ & $\begin{array}{c}\mathbf{K}_{\mathrm{IC}}\left(\mathbf{M P a m}^{1 / 2}\right) \\
( \pm \sigma=0,05)\end{array}$ \\
\hline I & 150 & G. vidriado & 5,1 & 6,40 & 1,25 \\
\hline II & 300 & & 3,5 & 6,45 & 1,85 \\
\hline & 450 & & 3,4 & 6,70 & 1,95 \\
\hline & 450 & & 3,5 & 6,70 & 1,90 \\
\hline & 600 & & 3,5 & 6,15 & 1,75 \\
\hline III & 750 & & 3,3 & 6,15 & 1,85 \\
\hline & 750 & & 2,8 & 6,00 & 2,20 \\
\hline & 900 & & 3,1 & 6,25 & 2,00 \\
\hline IV & 6000 & G. porcelánico & 2,7 & 6,00 & 2,30 \\
\hline V & $>12000$ & & 2,3 & 7,20 & 3,15 \\
\hline & $>12000$ & & 2,3 & 7,50 & 3,30 \\
\hline
\end{tabular}




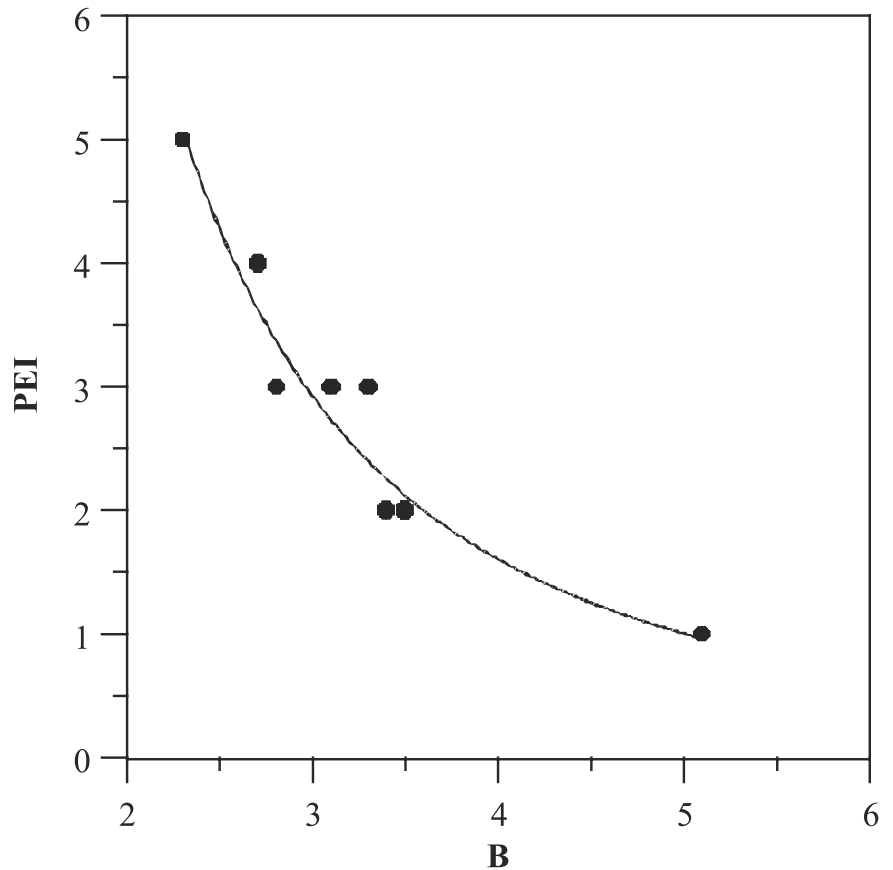

Figura 1. Representación de los valores de fragilidad (B) de una serie de pavimentos de monococción vidriados y de gres porcelánico comerciales en relación con la escala en que se clasifican después del ensayo PEI de resistencia a la abrasión. (La escala representada en el eje de ordenadas corresponde respectivamente a las escalas I hasta V del método PEI)

ña diferencia entre los valores extremos de la escala PEI (6). En cambio, cuando se usa la misma representación, pero frente al parámetro de fragilidad $\mathrm{B}$, se aprecia una mayor diferencia entre el comportamiento de los materiales aquí ensayados. Se comprueba tal y como ya se expuso en (11) que los valores de fragilidad (B) son menores para valores mayores de la escala PEI y que dicha variación sigue en los resultados que aquí se presentan una tendencia asintótica que se corresponde con la ecuación :

$\log \mathrm{y}=-2,06931 \log \mathrm{x}+3,34539$ (coeficiente de regresión $=0,953$ )

siendo y el grado de clasificación en la escala del método PEI y $x$ el valor de la fragilidad (B). La tendencia observada en este caso es del mismo tipo que la mostrada en (5) entre las revoluciones que soporta un material en el momento de perder su brillo cuando se relaciona con la rugosidad final obtenida y con el valor de tenacidad de fractura. En este sentido es preciso señalar que esta gráfica permite comprobar la existencia de una relación entre el coeficiente de fragilidad, tal y como aquí se ha definido, y el grado o escala de resistencia a la abrasión según el método PEI. De hecho, tendría más sentido físico la representación del número de revoluciones que soporta la superficie del material antes de la pérdida de brillo, y que corresponden a cada escala del método PEI, frente al parámetro B de fragilidad. Para ello, se ha realizado dicha representación teniendo en cuenta el número de revoluciones (5) de la plataforma giratoria del ensayo PEI. Se han ensayado diversos tipos de ajuste obteniéndose coeficientes de correlación muy bajos

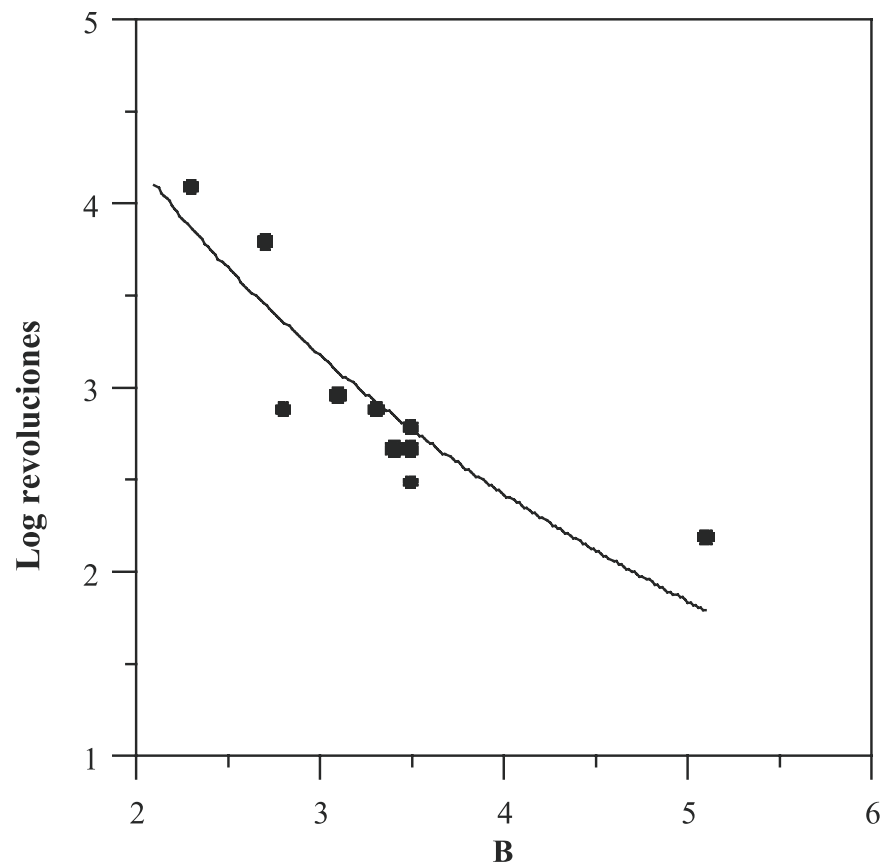

Figura 2. Representación de las revoluciones de la plataforma giratoria del ensayo PEI, antes de la pérdida de brillo de la superficie de la plaqueta cerámica, frente al valor de fragilidad (B) para un serie de pavimentos cerámicos vidriados y de gres porcelánico comerciales.

(entre 0,73 y 0,85 ) para ajustes lineales, exponenciales y logarítmicos. Se da en la Figura 2 el ajuste logarítmico con un coeficiente de regresión del 0,825. A pesar de este bajo valor del coeficiente en relación con el que se obtiene en la gráfica de la figura 1, se incluye dicha gráfica por su mayor sentido físico frente a la anterior en función de las escalas que indican las Normas EN 154 e ISO 10545/7.

Respecto a los valores de fragilidad calculados a partir de los resultados de $\mathrm{H}_{\mathrm{V}}$ y $\mathrm{K}_{\mathrm{IC}}$ dados por otros autores en vidriados comerciales muy semejantes a los aquí ensayados (4)(5), se ha comprobado que el intervalo de valores de fragilidad en nuestro caso alcanzan valores más altos que los de los citados autores, siendo el valor más bajo de fragilidad el que corresponde a un vidriado de tipo vitrocerámico aplicado por el método de "firestream" y que corresponde a una clase BI de la Norma Europea de clasificación general de pavimentos de tipo cerámico. Sin embargo, los valores aquí obtenidos se corresponden bien con los dados en (8) (11). Esta discrepancia puede ser debida a que en el caso de las referencias $(4,5)$ se ensayaron las indentaciones con cargas menores de $\mathrm{P}=4,91 \mathrm{~N}$, por lo que no serían en principio comparables dichos valores con los obtenidos en este trabajo. Aun así, a partir de datos bibliográficos y otros recogidos de investigaciones anteriores sobre materiales vítreos y vitrocerámicos (12-15) se ha calculado asimismo el parámetro de fragilidad, comprobándose que se alcanzan valores dentro del intervalo aquí encontrado para materiales de pavimentos y revestimientos cerámicos comerciales e incluso otros anteriormente obtenidos por Rincón y Romero (12) e incluso en el caso de uno masivo de tipo vitrocerámico como es 
el Neoparis ${ }^{\circledR}$ en el que para esta investigación se ha determinado su valor de fragilidad (Tabla II). El módulo de elasticidad, E, se ha obtenido a partir de las indentaciones Knopp tal y como se describe por Rincón y Capel para vidrios del sistema $\mathrm{Li}_{2} \mathrm{O}-\mathrm{SiO}_{2}$ (10) a partir de las formulaciones de Marshall y Lawn (3). Muy recientemente, se ha aplicado este mismo concepto de fragilidad en el caso de recubrimientos sol-gel parcialmente densificados de circona, sílice y del sistema de composición $\mathrm{ZrO}_{2}-\mathrm{SiO}_{2}$ obtenidos sobre sustratos de vidrio, permitiendo este método una diferenciación de propiedades mecánicas entre una amplia gama de materiales de recubrimiento (16).

Dado que no se observa en las medidas aquí realizadas una relación lineal entre el índice de fragilidad y los valores PEI, se ha recurrido también a introducir el efecto del comportamiento elástico del material con el valor de índice de fragilidad corregida : $\mathrm{B}^{*}=\mathrm{B} \times \mathrm{E}$. Aun así, la introducción de dicha fragilidad corregida multiplicando el parámetro de fragilidad por el valor de módulo de elasticidad no ha dado lugar a una mayor diferenciación entre los materiales ensayados en esta investigación. Sin embargo, si se calcula la relación : B* $=B$ / $\mathrm{E}$, tal y como se muestra en la Figura 3 se aprecian mejor las diferencias de comportamiento entre los diferentes recubrimientos que quedaban solapados en la misma escala de PEI en la representación anterior. En este caso la ecuación que se ajusta a dicha tendencia sería:

$$
y=-2,11537 \log x+4,22599 \text { (coeficiente de regresión }=0,93 \text { ) }
$$

Actualmente, se realizan observaciones microestructurales por microscopía electrónica de barrido (SEM) con el fin de relacionar estos resultados con la microestructura tanto de los pavimentos aquí considerados como de otro tipo de materiales en los que se tiene ya propia experiencia de su comportamiento por ensayos de indentación.

\section{CONCLUSION}

Se propone un nuevo método para evaluar la resistencia a la abrasión de pavimentos y revestimientos cerámicos tanto si se han recubierto con vidriados como si su superficie es no vidriada y pulida. Consiste dicho método en la determinación por ensayos de indentación, tanto Vickers como Knopp del parámetro o índice de fragilidad (B), que se define como el cociente entre los valores de microdureza Vickers y de tenacidad de fractura. Este sencillo método permitiría una mejor evaluación de la resistencia a la abrasión que el método tradicionalmente aceptado por el sector industrial para este tipo de materiales que la que se utiliza habitualmente según el método PEI. Se han contrastado los resultados obtenidos con los valores que se obtienen para una amplia gama de productos, comprobándose que existe una correlación entre el parámetro de fragilidad y la resistencia a la abrasión. Actualmente se investiga en más profundidad el sentido físico y la formulación de este índice de fragilidad en relación con la microestructura de los pavimentos de tipo cerámico.

\section{AGRADECIMIENTOS}

Los autores agradecen a la empresa FRITTA SL (Jordi Marco) por el suministro de muestras para esta investigación.
TABLA II. VALORES DE FRAGILIDAD DEDUCIDOS POR MEDIDAS DE INDENTACIÓN EN MATERIALES VITREOS Y VITROCERAMICOS OBTENIDOS EN ANTERIORES INVESTIGACIONES (12-15)

\begin{tabular}{|c|c|c|c|c|}
\hline material & $\begin{array}{c}\mathrm{B}\left(\mu \mathrm{m}^{-1 / 2}\right) \\
( \pm \sigma=0,05)\end{array}$ & $\begin{array}{c}\mathrm{H}_{\mathrm{v}}(\mathrm{GPa}) \\
( \pm \sigma=0,1)\end{array}$ & $\begin{array}{c}\mathrm{K}_{\mathrm{IC}}\left(\mathrm{MPam}^{1 / 2}\right) \\
( \pm \sigma=0,1)\end{array}$ & $\begin{array}{c}\text { E (GPa) } \\
( \pm \sigma=10)\end{array}$ \\
\hline \multirow[t]{3}{*}{$\begin{array}{l}\text { vidrios a partir de } \\
\text { cenizas volantes (12) }\end{array}$} & 3,83 & 6,9 & 1,8 & 101 \\
\hline & 3,57 & 5,0 & 1,4 & 59 \\
\hline & 3,11 & 5,3 & 1,7 & 61 \\
\hline $\begin{array}{c}\text { vitrocerámicos } \\
\text { Neoparis }^{\circledast}\end{array}$ & 2,78 & 5,0 & 1,8 & \\
\hline \multirow[t]{6}{*}{$\begin{array}{c}\text { Gres porcelánico } \\
\text { modificado }\end{array}$} & 4,69 & 6,1 & 1,3 & 71 \\
\hline & 3,10 & 6,2 & 2,0 & 247 \\
\hline & 2,73 & 6,3 & 2,3 & 305 \\
\hline & 2,53 & 7,1 & 2,8 & 165 \\
\hline & 2,27 & 7,5 & 3,3 & 232 \\
\hline & $2,1-3,0$ & $6,0-7,5$ & $2,0-3,5$ & $150-300$ \\
\hline
\end{tabular}

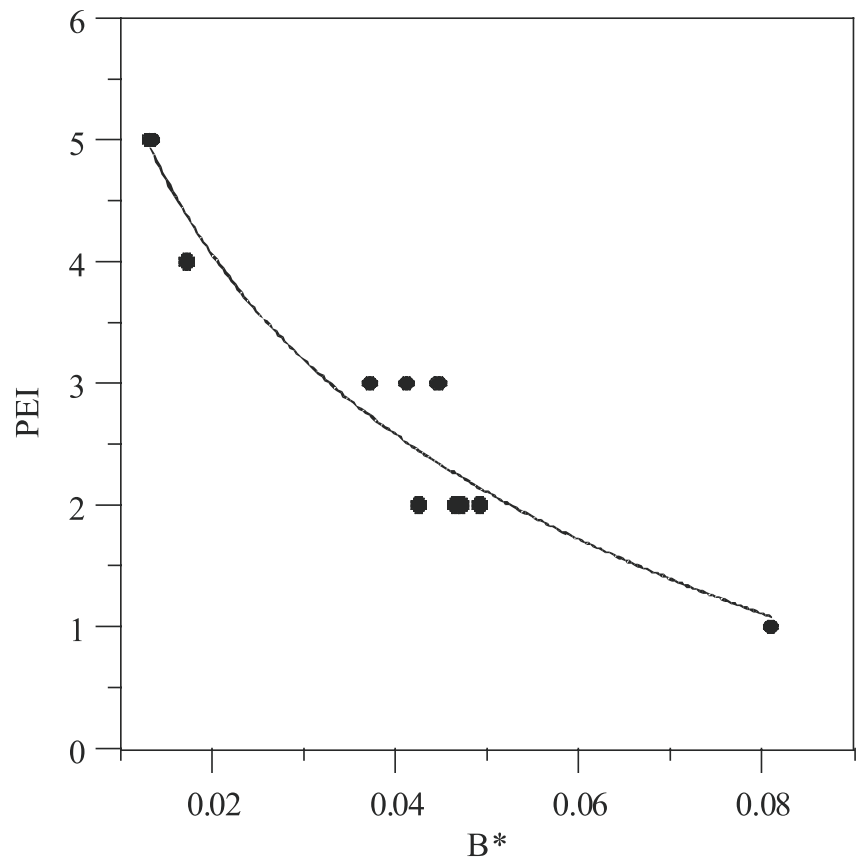

Figura 3. Representación de los valores de fragilidad corregida (B* $=\mathrm{B} / \mathrm{E}$ ) para un serie de pavimentos cerámicos vidriados y de gres porcelánico comerciales en relación con la escala en que se clasifican después del ensayo PEI de resistencia a la abrasión. (La escala representada en el eje de ordenadas corresponde respectivamente a las escalas I hasta V del método PEI)

\section{REFERENCIAS}

1. Normas EN 154 y ISO10545/7. Resistencia a la abrasión superficial. Baldosas esmaltadas.

2. E. Enrique Navarro, Aplicaciones de los materiales cerámicos, vidrios y composites en la construcción, En : Aplicaciones de los Materiales Cerámicos, Vidrios y Composites en la Construcción, Eds. J. Ma. Rincón, J. P. Gutierrez y M. Romero, Inst. E. Torroja, CSIC, Madrid, 1996,p. 43-49.

3. R. Lawn and D. B. Marshal, Hardness, Toughness and Brittleness: An Indentation Analysis, J. Amer. Ceram. Soc. 62 [7-8] 347-350 (1979)

4. A. Moreno, E. Montiel, J. Toledo y A. Escardino, Relación entre las propiedades mecánicas de vidriados cerámicos y su comportamiento al someterlos a tensiones externas, Ceramica Información 195,2-9 (1994)

5. A. Escardino, A. Moreno, M ${ }^{a}$. J. Ibañez y A. Barba, Relación entre las propiedades mecánicas y su resistencia al desgaste, Bol. Soc. Esp. Ceram. Vidr. 39 (2) 209-214 (2000).

6. L. Esposito y A. Tucci, Abrasión de Superficies de Azulejos Cerámicos Esmaltados, Bol. Soc. Esp. Ceram. Vidr. 39 165-171(2000) . 
7. A.R. Bocccaccini, Assesment of brittlenes in glass- ceramics and particulate glass matrix composites by indentation data, J. Mats. Sci. 15,11191121(1996).

8. A. R. Boccaccini, The Relationship between Wear Behaviour and Brittleness Index in Engineering Ceramics and Dispersion- Reinforced Composites, Interceram 48[3]176-187(1999)

9. J. Sehgal, Y. Nakao, H. Takahashi and S. Ito, J. Mater. Sci. Lett.14,167 (1995)

10. J. $\mathrm{M}^{\mathrm{a}}$ Rincón and F. Capel, Microindentation behaviour, $\mathrm{K}_{\mathrm{IC}}$ factor determination and microstructure analysis of some $\mathrm{Li}_{2} \mathrm{O}-\mathrm{SiO}_{2}$ glass- ceramic materials. Ceramic International 11(3) 97-102 (1985).

11. A. R. Boccaccini, Comment on "Surface Abrasión of Glazed Tiles", Bol. Soc. Esp. Ceram. Vidr. 40[4] 309-310 (2001)

12. L. Barbieri, T. Manfredini, I. Queralt, J. Ma. Rincón and M. Romero, Glass Technology 38(5)165-17 (1997)
13. M. Romero and J.Ma. Rincón, The controled vitrification/ crystallization process applied to the recycling of inorganic industrial wastes, Bol. Soc. Esp Ceram. Vidr. 39 (1) 155-163 (2000)

14. T. Manfredini, M. Romagnoli and J.Ma. Rincón, Porcelainized stoneware: architectural, processing and physico/ mechanical properties, Materiales de Construcción 46 (242- 243) 107- 118 (1996)

15. Ma . S. Hernández and J. Ma. Rincón, New Porcelainized Stoneware Materials Obtained By Recycling Of MSW Incinerator Fly Ashes and Granite Sawing Residues, Ceramics International 27,713-720 (2001)

16. Ma . A. Villegas, J. Ma. Rincón, M. García- Heras y M. Romero, Indentación y fragilidad de recubrimientos sol- gel parcialmente densificados, En: III Congreso Nac. de Propiedades Mecánicas de Sólidos, PMS 2002, Gandía, De. V. Amigó, Univ. Politécnica de Valencia, 2002, p. 715-719

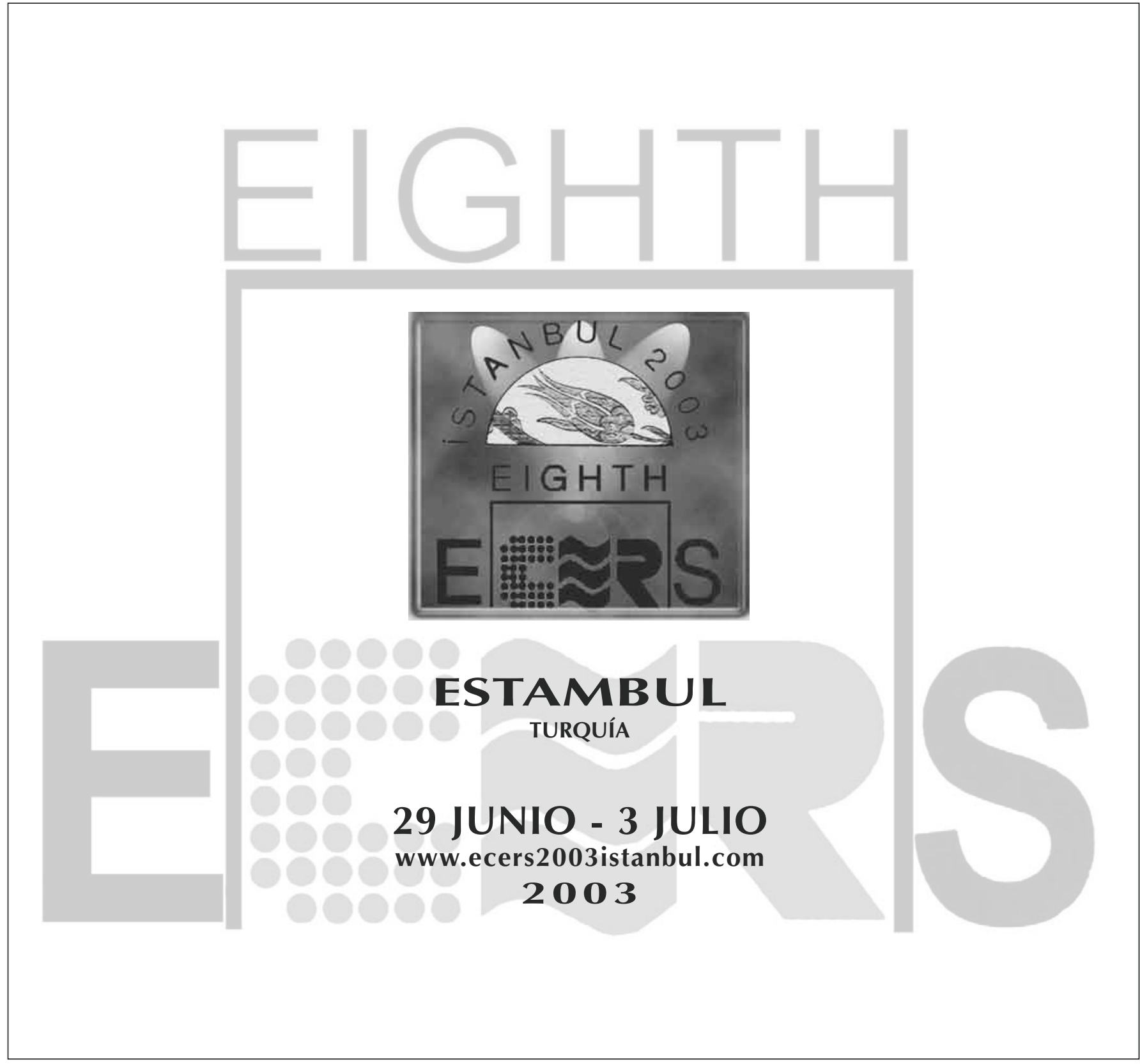

